\title{
Including Socioeconomic Status in Coronary Heart Disease Risk Estimation
}

\author{
Peter Franks, $M D^{1,2}$ \\ Daniel J. Tancredi, $\mathrm{PbD}^{2,3}$ \\ Paul Winters, $M S^{4}$ \\ Kevin Fiscella, MD, MPH \\ ${ }^{1}$ Center for Healthcare Policy and Research, \\ Sacramento, California \\ ${ }^{2}$ Department of Family \& Community \\ Medicine, University of California, Davis, \\ Sacramento, California \\ ${ }^{3}$ Department of Pediatrics, University of \\ California at Davis, Sacramento, California \\ ${ }^{4}$ Department of Family Medicine, Univer- \\ sity of Rochester, Rochester, New York \\ ${ }^{5}$ Departments of Family Medicine, Com- \\ munity \& Preventive Medicine, Oncology, \\ University of Rochester, Rochester, \\ New York

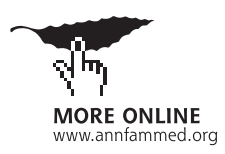

Conflicts of interest: none reported

\section{CORRESPONDING AUTHOR}

Peter Franks, MD

Department of Family \& Community

Medicine

University of California at Davis

4860 Y St, Suite 2300

Sacramento, CA, 95817

pfranks@ucdavis.edu.

\begin{abstract}
PURPOSE Socioeconomic status (SES) predicts coronary heart disease independently of the Framingham risk-scoring factors included in cholesterol treatment guidelines, possibly resulting in undertreatment of lower SES persons. We examined whether hybrid SES measures (based on area measures of income and individual education) address this bias and derived an approach to incorporating SES information into treatment guidelines.
\end{abstract}

METHODS The Atherosclerosis Risk in Communities study data (initiated in 1987 with a 10-year follow-up of 15,495 adults aged 45 to 64 years in 4 southern and midwestern communities) were used to assess the calibration bias of 4 Cox models predicting 10-year coronary heart disease risk: Framingham risk score alone, and Framingham risk score plus SES using an individual-based measure (income less than 150\% federal poverty level or less then 12 years of schooling), and 2 hybrid SES measures substituting area-based income measures (block group or zip code median incomes of less than 25th national percentiles) for the individual income component. Revised cholesterol treatment thresholds based on SES risk were also derived.

RESULTS Use of either the block group hybrid or individual-based SES measures eliminated the significant SES bias observed using Framingham risk score alone. Cholesterol treatment guideline thresholds of $10 \%$ and $20 \%$ coronary heart disease risk (based on the Framingham risk score) were reduced to $6 \%$ and $13 \%$ for those with low SES.

CONCLUSIONS Using patient income based on block group and individual education minimizes the SES bias in Framingham risk scoring and suggests more aggressive cholesterol treatment thresholds for low-SES persons.

Ann Fam Med 2010;8:447-453. doi:10.1370/afm.1167

\section{INTRODUCTION}

$\mathrm{F}$ ramingham risk scoring underestimates coronary heart disease risk for persons of lower socioeconomic status (SES), because SES is an independent risk factor not included in Framingham risk scoring. ${ }^{1,2}$ Consequent inappropriately high treatment thresholds for low-SES persons may contribute to worsening SES disparities in coronary heart disease. ${ }^{3} \mathrm{We}$ have previously found that incorporating an individual-based SES measure (using individual patient education and household income) into coronary heart disease risk assessment mitigates underestimation of coronary heart disease risk among low SES persons determined by the Framingham risk score alone. ${ }^{2}$ That approach, however, required knowledge of patients' household income and family size, and their conversion into a percentage of the federal poverty level. Clinicians may be reluctant to ask patients about income; patients may be reluctant to disclose the information. In addition, no approach to incorporating SES risk into risk-based cholesterol treatment decision making was provided. Here we explore alternative approaches to assessing SES risk (using the patient's place of residence) and derive an approach to incorporating SES risk into treatment guidelines. 
Area-based SES measures (using the patient's place of residence) have been used as proxies for individual measures in diverse situations. ${ }^{4-8}$ Indeed, area-based measures capture unique aspects of coronary heart disease risk beyond individual SES measures. ${ }^{9}$ Areabased measures can be developed by linking the patient's address to census data for that area of residence. Such measures are currently used in the United Kingdom to account for SES in coronary heart disease risk stratification ${ }^{10}{ }_{i}$ however, the performance of area measures for this purpose in the United States is unknown.

First, we evaluated whether substituting area-based measures of income (based on block group-the smallest geographic area for which the US census publishes sociodemographic information - or zip code median income) for the individual income measure would enable derivation of a hybrid SES measure comparable to the individual-based SES measure.

Second, we derived a method of incorporating SES into the National Cholesterol Education Program's Detection, Evaluation and Treatment of High Blood Cholesterol in Adults (Adult Treatment Panel III) cholesterol treatment decision guidelines without revising the Framingham risk score or the framework of these treatment guidelines.

\section{METHODS}

\section{Sample}

The Atherosclerosis Risk in Communities study is a prospective investigation, conducted in 4 US communities, designed to examine the natural history of atherosclerosis and related diseases. ${ }^{11}$ The Atherosclerosis Risk in Communities cohort component was initiated in 1987. Each Atherosclerosis Risk in Communities field center randomly selected and recruited samples of approximately 4,000 individuals aged 45 to 64 years in each of the following communities: Forsyth County, North Carolina (75\% urban), Jackson, Mississippi (100\% urban), Minneapolis suburbs, Minnesota (100\% urban), and Washington County, Maryland (57\% urban). In 1987-1989 participant examinations yielded medical and sociodemographic data. Roughly $75 \%$ of eligible respondents participated in the baseline interview, and $80 \%$ of these participated in the baseline examination. ${ }^{12}$ Data regarding coronary heart disease events were collected through annual telephone interviews, follow-up examinations, surveys of hospital discharge data, and death certificates from state vital statistics offices. ${ }^{11}$ We excluded participants who reported coronary heart disease or equivalents (stroke, peripheral vascular disease, or diabetes) at baseline.

\section{Socioeconomic Status}

We derived a dichotomous, individual-based measure of SES using income and education. We defined participants to be of lower SES if they had fewer than 12 years of schooling or had household incomes of less than $\$ 12,000$ (corresponding to $150 \%$ of the federal poverty level for an average household in 1987). ${ }^{13}$

We derived median income of the participant's place of residence using 1990 US census data, aggregated at the block group or zip code levels. Participant addresses were mapped to census block group, enabling derivation of the median household income for the census block containing the participant's address. Using national reference points, we identified quartiles of block group and zip code median household incomes. To derive the hybrid SES measures paralleling the individual measure, we identified participants to be of lower SES if they had fewer than 12 years of schooling or lived in a block group (block group-based hybrid SES) or zip code (zip code-based hybrid SES) in the bottom national quartile of household median income.

\section{Framingham Risk Scoring}

We used Framingham risk scoring to derive the 10year risk for a coronary heart disease event or death for men and women as proposed in the National Cholesterol Education Program..$^{14}$ Framingham risk scoring uses participant age, sex, total cholesterol, high-density lipoprotein cholesterol, smoking status, systolic and diastolic blood pressure, use of antihypertensive agents, family history of premature coronary heart disease, and coronary heart disease or equivalent (stroke and diabetes).

\section{Outcome}

We assessed the timing of any coronary heart disease event. Participants not observed to develop coronary heart disease within 10 years were considered censored (or when follow-up ended, if sooner). Subscribing to the Atherosclerosis Risk in Communities study criteria, we based the diagnosis of incident coronary heart disease on electrocardiographic and cardiac enzyme criteria or death certificate data and arbitrated by an Atherosclerosis Risk in Communities physician panel. ${ }^{15}$

\section{Analyses}

Data were analyzed using Stata analytic software (version 11.0, StataCorp, College Station, Texas). We conducted Cox proportional hazards regression analyses, with survival time as the time to event or censoring. To increase precision, models reported here included both sexes. Initial models included the individual's Framingham risk score of estimated risk, sex, the SES 
measures, and all 2-way interactions. None of the effects for sex or interactions was statistically significant, so these terms were dropped from the final models. The proportional hazards assumption was supported in every analysis.

Prediction models included (1) Framingham risk score alone, (2) Framingham risk score plus individual-based SES measure, (3) Framingham risk score plus block group-based hybrid SES measure, and (4) Framingham risk score plus zip code-based hybrid SES measure. We used the Martingale residuals from each model to assess the calibration of coronary heart disease event risk by the dichotomous SES indicators (both hybrid and individually based) not included in the model. Details of the analysis can be found in the

in annfammed.org/cgi/content/full/8/5/447/DC1. Cali-

bration is the extent to which the model correctly predicts the outcome (here incident coronary heart disease) by group membership (here SES).

To better reflect the influence of the Framingham risk score in the Cox models, the Framingham risk score (on a probability scale) was modeled as its complementary log-log transform $(\log [-\log (1-$ Framingham risk score $)]$ ). We also derived Kaplan-Meier survival curves and survivor functions adjusted for Framingham risk score.

We conducted sensitivity analyses stratifying by sex, substituting the 20th and 30th percentiles for areabased median income for the 25 th percentile, using income and education measure alone instead of the combined SES measures, and using coronary heart disease mortality rather than coronary heart disease event as the outcome. A further analysis examined the calibration of using block group income data when it was available, but using zip code income when it was not.

Finally, we derived a simple method of incorporating SES risk into treatment decisions (detailed in the Supplemental Appendix).

\section{RESULTS}

There were 15,732 participants in Atherosclerosis Risk in Communities study. Excluding the $15 \%$ of participants with baseline coronary heart disease or equivalents and the less than $1 \%$ of participants with missing Framingham risk score data elements resulted in a study sample of 12,945 . Because of missing individual SES information (primarily income), there were 12,393 persons in the individual-based SES model $(3,418$, or $28 \%$, in the lower SES category). Block group address mapping accomplished for $91 \%$ of the total sample yielded 12,032 persons in the block group-based hybrid SES model $(4,243$, or $35 \%$, in the lower SES category). Zip code-based median income data were available for $99.9 \%$, resulting in 12,921 persons in the zip code-based hybrid SES model $(3,378$, or $26 \%$, in the lower SES category).

Descriptive sociodemographic characteristics of the sample are shown in Table 1 . The study sample overrepresents those in the higher national household income quartiles.

In Table 2 are displayed the results of the 4 Cox survival models. Adding the SES measures to the survival models had little effect on the Framingham risk score hazard ratios (comparing upper and lower). The SES hazard ratios for both the individual-based and block group-based hybrid SES measures were significant and similar and slightly greater than for the zip code-based hybrid SES measure. Supplemental Table 1, available online at http://annfammed.org/cgi/ content/full/8/5/447/DC1, shows that the hazard ratios were similar for men and women in analyses stratified by sex.

Figure 1 illustrates the impact of SES on coronary heart disease risk. The top 2 Kaplan-Meier curves show the similar effects of individual-based and block group-based hybrid SES. The lower survivor functions, adjusted for Framingham risk score, again show that the 2 SES methods exert similar, albeit smaller, effects.

Supplemental Table 2, available online at http:// annfammed.org/cgi/content/full/8/5/447/DC1, summarizes the calibration analyses with the survival model using Framingham risk score alone; SES calibration bias when assessed by all SES measures was

\begin{tabular}{lc}
\hline $\begin{array}{l}\text { Table } 1 . \text { Sociodemographic } \\
\text { of the Study Sample }\end{array}$ \\
\hline Characteristic & Value \\
\hline Eligible sample, No. & 12,945 \\
Age, mean years (SD, range) & $53.9(5.7,45-64)$ \\
Female, \% & 56.2 \\
Black, \% & 23.5 \\
$<12$ years education, $\%$ & 21.1 \\
$<\$ 12,000$ income, $\%$ & 13 \\
Quartiles of national median house- & \\
hold zip code income, $\%$ & \\
$<\$ 20,230$ & 8.9 \\
$\$ 20,230-<\$ 25,200$ & 14.0 \\
$\$ 25,200-<\$ 32,266$ & 30.6 \\
$>\$ 32,266$ & 46.5 \\
Quartiles of national median house- & \\
hold block group income, $\%$ & \\
$<\$ 20,463$ & 16.4 \\
$\$ 20,463-<\$ 27,857$ & 16.7 \\
$\$ 2,7857-<\$ 38,250$ & 32.1 \\
$>\$ 38,250$ & 34.8 \\
\hline
\end{tabular}

Notes: Eligible sample includes participants without baseline coronary heart disease stroke, peripheral vascular disease, or diabetes, and no missing Framingham risk score elements. 
significant $(P<.001)$. Coronary heart disease prediction was overestimated by $17.5 \%$ in those with higher individual-based SES and underestimated by $24 \%$ in those with lower individual-based SES, calibration bias assessed by block group-based hybrid SES calibration was similar.

Supplemental Table 3, available online at

il http://annfammed.org/cgi/content/full/8/5/447/

DC1, summarizes the calibration analyses with the
3 survival models using Framingham risk score and SES adjustments. SES calibration bias was reduced when assessed by the SES measures not in the model.

The SES calibration bias for both individual-based and block group-based hybrid SES prediction models was not clinically or statistically significant. The SES calibration bias in the zip code-based hybrid SES model was reduced (when assessed by the SES measures not in the model), but remained statistically significant.

Table 2. Cox Survival Analyses of Coronary Heart Disease Risk Prediction Using Framingham Risk Scoring SES Adjustment and With Individually Based and Hybrid SES Adjustment

\begin{tabular}{|c|c|c|c|c|c|c|}
\hline \multirow[b]{2}{*}{ Survival Analysis } & \multicolumn{2}{|c|}{$\begin{array}{l}\text { Individual-Based SES } \\
\qquad(\mathrm{n}=12,393)\end{array}$} & \multicolumn{2}{|c|}{$\begin{array}{l}\text { Block Group-Based Hybrid SES } \\
\qquad(\mathrm{n}=12,032)\end{array}$} & \multicolumn{2}{|c|}{$\begin{array}{l}\text { Zip Code-Based Hybrid SES } \\
\qquad(n=12,921)\end{array}$} \\
\hline & $\begin{array}{l}\text { Hazard Ratio } \\
(95 \% \mathrm{Cl})\end{array}$ & $P$ Value & $\begin{array}{l}\text { Hazard Ratio } \\
(95 \% \mathrm{Cl})\end{array}$ & $P$ Value & $\begin{array}{l}\text { Hazard Ratio } \\
(95 \% \mathrm{Cl})\end{array}$ & $P$ Value \\
\hline \multicolumn{7}{|c|}{ Framingham risk score alone } \\
\hline Framingham risk score & $2.34(2.12-2.57)$ & $<.01$ & $2.29(2.08-2.52)$ & $<.01$ & $2.33(2.12-2.55)$ & $<.01$ \\
\hline \multicolumn{7}{|c|}{ Framingham risk score + SES } \\
\hline Framingham risk score & $2.30(2.09-2.53)$ & $<.01$ & $2.25(2.04-2.47)$ & $<.01$ & $2.30(2.09-2.52)$ & $<.01$ \\
\hline Lower SES ${ }^{a}$ & $1.60(1.34-1.92)$ & $<.01$ & $1.58(1.32-1.89)$ & $<.01$ & $1.42(1.18-1.70)$ & $<.01$ \\
\hline
\end{tabular}

SES = socioeconomic status.

Note: Framingham risk score is complementary log-log transformed: log[-log (1-Framingham risk score)].

a Individually based, block group-based hybrid, or zip code-based hybrid SES adjustment.

Figure 1. Kaplan-Meier and Framingham risk score-adjusted survival curves for coronary heart disease event, by individual-based and block group-based hybrid SES.
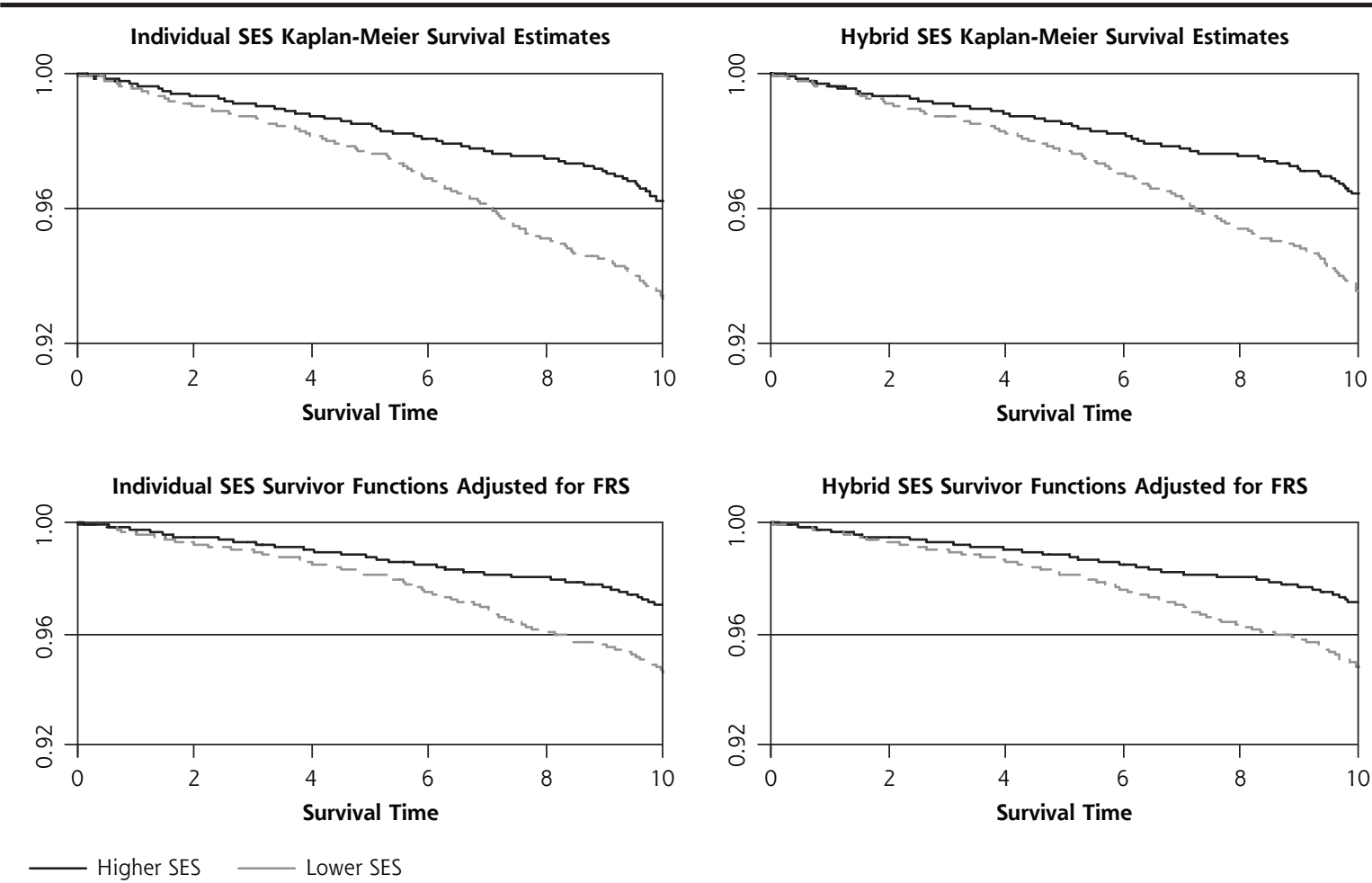

FRS = Framingham risk score; SES = socioeconomic status. 
Sensitivity analyses examined coronary heart disease mortality only and by substituting the 20th and 30th percentiles as thresholds for the area-based income measures. The results of all these analyses were consistent with those presented. Analyses using education or individual- or area-based income measures alone did not eliminate the significant SES bias in coronary heart disease prediction. The strategy of using block group information when available, but zip code information when it was not, also eliminated the SES bias in coronary heart disease prediction.

The hazard ratios for lower SES (individual-based or block group-based hybrid) mean that the nominal coronary heart disease thresholds of $10 \%$ and $20 \%$ predicted by the Framingham risk score are reached for lower SES persons when their predicted coronary heart disease risks are $6 \%$ and $13 \%$, respectively.

Coronary heart disease risk predicted by the Framingham risk score among those with more than 2 risk factors was more likely among lower than higher SES persons. Overall, only 3\% of those with more than 2 risk factors had a predicted coronary heart disease risk of greater than $10 \%$ (the lower Adult Treatment Panel III threshold). Among lower SES persons, however, $5 \%$ of those with more than 1 risk factor had a predicted coronary heart disease risk above $6 \%$ (the proposed modified lower treatment threshold for lower SES persons.

\section{DISCUSSION}

Block group-based hybrid and individual-based SES measures eliminated and the zip code-based SES reduced the SES bias in coronary heart disease risk estimation resulting from using Framingham risk scoring alone. Further, current Adult Treatment Panel III cholesterol treatment guidelines recommend Framingham risk scoring only when 2 or more risk factors are present (since there is a low probability of coronary heart disease with fewer than 2 risk factors). To be consistent with these guidelines, the current analyses suggested that the Framingham risk score should be calculated for lower SES persons if they have 1 or more risk factors. Finally, cholesterol treatment risk thresholds of $10 \%$ and $20 \%$ should be lowered to $6 \%$ and $13 \%$ for lower SES persons.

These findings represent the first US analyses on the potential for using area-based SES in coronary heart disease risk estimation. Findings from the United Kingdom have shown that cardiovascular risk tools that include area-based SES mitigate SES bias in risk assessment. ${ }^{1,16,17}$ Though area and individual measures capture different aspects of SES, ${ }^{9}$ the hybrid block group-based measure appeared to capture the entire
SES hazard associated with the individual-based SES measure.

The zip code-based hybrid measure reduced, but did not eliminate, the SES bias. This measure is easier to derive than the block group-based measure, since it does not require address mapping and generates fewer missing data.

We developed an approach to incorporating SES into cardiac risk stratification based on the well-validated and familiar Framingham risk score. The modifications should be easy for clinicians to accommodate. In the United Kingdom, by contrast, the QRISK and ASSIGN systems incorporate SES risk directly into the risk calculation, completely replacing the Framingham risk scoring ${ }^{1,10} ;$ a user-friendly tool for estimating cardiovascular risk incorporating patient SES based on UK postal codes is available (http://www.qrisk.org).

The study has notable strengths and limitations. The results are based on a diverse cohort of middleaged persons free of coronary heart disease at baseline. Coronary heart disease risk factors and SES were assessed at baseline; coronary heart disease events were prospectively assessed using predefined criteria. Misclassification of SES resulting from incorrect addresses would tend to bias results toward the null.

As with most longitudinal studies of coronary heart disease (including Framingham), this study had limited power to assess the impact on women apart from men; women have lower rates of coronary heart disease. We found no evidence of significant interactions between sex and SES or Framingham risk score on coronary heart disease risk, and analyses stratified by sex yielded consistent results.

The use of dichotomies to describe SES risk is an oversimplification because SES exerts its effects in a graded fashion. We used dichotomies to enable development of clinically manageable cut points.

In this study, address mapping and income data collection were incomplete. When data are missing, alternative measures of SES will be needed (such as insurance status, occupation, zip code SES data, or housing information). The strategy of using block group information or, if not available, zip code information, minimized miscalibration when tested using the individual-based SES measure.

We found the area-based SES used was robust to different choices of cut point for identification of low income, and to restricting the outcome to death from coronary heart disease. It would be helpful, however, to validate the findings in an independent data set and with different census data to determine whether using the 25 th percentile provides a stable estimate of SES risk for coronary heart disease over time.

The method proposed for using area-based income 
measures avoids some of the challenges of deriving an individual-based income measure (primarily privacy concerns and the need to convert household income information into a percentage of the federal poverty level). But the area-based method presents technical problems (requiring mapping patients' addresses). ${ }^{18}$ The advent of electronic medical records could facilitate automatic generation of this information; development of such systems will require sufficient consensus that the approach outlined here is warranted. Before such systems become available, physicians could obtain area-based income information for their area by downloading the information from the US Census Web site, commercial vendors, or local academic institutions. Physicians could also develop local maps highlighting low-income block group or zip code areas (such as those used to indicate water fluoridation levels to determine appropriate supplementation).

These findings have potentially important clinical implications for reducing coronary heart disease disparities. ${ }^{19}$ As with other sociodemographic risk factors, such as age and sex, the relationship between SES and coronary heart disease risk is likely complex. Emerging evidence points to lifelong material, psychosocial, and behavioral pathways that affect aging-related processes. ${ }^{20,21}$ Although the concept of treating SES-related risk may seem misdirected at first, minimizing the disparity in coronary heart disease will likely require a multipronged approach that addresses both current, remediable, intervening risk factors, eg, behavioral risk factors, health care access, and affordability, in addition to use of pharmacotherapy based on realistic estimates of coronary heart disease risk. ${ }^{22}$ Even though our analyses do not address differences in SES-based statin use, ${ }^{23}$ the growing availability of low-cost statins may promote diffusion to lower SES groups. ${ }^{24}$

It is unknown whether lower treatment thresholds for lower SES persons will reduce growing SES disparities in coronary heart disease. It is unlikely that randomized trials will be conducted to address this question. Furthermore, it will be difficult to conduct appropriate new observational studies on coronary heart disease risks in an era of widespread use of statins. The findings suggest, however, a plausible, low-risk clinical strategy absent more definitive information. Patients who either did not graduate from high school or live in a low-income area (or have low household income) with Framingham risk scores of $6 \%$ or $13 \%$ should be managed as if their Framingham risk score was $10 \%$ or $20 \%$. Consistent with Adult Treatment Panel III guidelines, lower SES persons should have their Framingham risk scores calculated if they have 1 or more risk factors. Incorporating SES risk into coronary heart disease risk stratification will reduce the SES bias involved in using the Framingham risk score alone. In turn, such an approach to the management of coronary heart disease risk may help reduce the growing socioeconomic disparity in coronary heart disease.

To read or post commentaries in response to this article, see it online at http://www.annfammed.org/cgi/content/full/8/5/447.

Key words: Coronary disease/prevention \& control; cholesterol; epidemiology; risk factors

Submitted December 7, 2009; submitted, revised, March 20, 2010; accepted April 1, 2010.

Funding support: This study was supported by the National Heart Lung and Blood Institute, grant No. 1R01 HL081066-01A2.

Results of this study were presented at the Annual Meeting of the North American Primary Care Research Group, November 15, 2009, Montreal, Canada.

\section{References}

1. Woodward M, Brindle P, Tunstall-Pedoe H. SIGN group on risk estimation. Adding social deprivation and family history to cardiovascular risk assessment: the ASSIGN score from the Scottish Heart Health Extended Cohort (SHHEC). Heart. 2007;93(2):172-176.

2. Fiscella K, Tancredi D, Franks P. Adding socioeconomic status to Framingham scoring to reduce disparities in coronary risk assessment. Am Heart J. 2009;157(6):988-994.

3. Singh GK, Siahpush M. Increasing inequalities in all-cause and cardiovascular mortality among US adults aged 25-64 years by area socioeconomic status, 1969-1998. Int J Epidemiol. 2002;31(3): 600-613.

4. Krieger N. Overcoming the absence of socioeconomic data in medical records: validation and application of a census-based methodology. Am J Public Health. 1992;82(5):703-710.

5. Fremont AM, Bierman AS, Wickstrom SL, et al. Use of indirect measures of race/ethnicity and socioeconomic status in managed care settings to identify disparities in cardiovascular and diabetes care quality. Health Aff. 2005;24(2):516-526.

6. Fiscella K, Franks P. Impact of patient SES on physician profiles: a comparison of census derived and individual measures. Med Care. 2001;39(1):8-14

7. Whitsel EA, Rose KM, Wood JL, Henley AC, Liao D, Heiss G. Accuracy and repeatability of commercial geocoding. Am J Epidemiol. 2004;160(10):1023-1029.

8. Krieger N, Chen JT, Waterman PD, Rehkopf DH, Subramanian SV. Race/ethnicity, gender, and monitoring socioeconomic gradients in health: a comparison of area-based socioeconomic measures-the public health disparities geocoding project. Am J Public Health. 2003;93(10):1655-1671.

9. Diez-Roux AV, Merkin SS, Arnett D, et al. Neighborhood of residence and incidence of coronary heart disease. N Engl J Med. 2001;345(2):99-106.

10. Hippisley-Cox J, Coupland C, Vinogradova Y, et al. Predicting cardiovascular risk in England and Wales: prospective derivation and validation of QRISK2. BMJ. 2008;336(7659):1475-1482.

11. The ARIC investigators. The Atherosclerosis Risk in Communities (ARIC) Study: design and objectives. Am J Epidemiol. 1989;129(4): $687-702$. 
12. Jackson R, Chambless LE, Yang K, et al. Differences between respondents and nonrespondents in a multicenter communitybased study vary by gender ethnicity. The Atherosclerosis Risk in Communities (ARIC) Study Investigators. J Clin Epidemiol. 1996;49(12):1441-1446.

13. US Census Bureau. Poverty Thresholds: 1987. http://www.census.gov/ hhes/www/poverty/threshld/thresh87.html.

14. Expert Panel on Detection, Evaluation, and Treatment of High Blood Cholesterol in Adults. Executive summary of the third report of the National Cholesterol Education Program (NCEP). JAMA. 2001;285(19):2486-2497.

15. Chambless LE, Folsom AR, Sharrett AR, et al. Coronary heart disease risk prediction in the Atherosclerosis Risk in Communities (ARIC) study. J Clin Epidemiol. 2003;56(9):880-890

16. Hippisley-Cox J, Coupland C, Vinogradova Y, Robson J, May M Brindle P. Derivation and validation of QRISK, a new cardiovascular disease risk score for the United Kingdom: prospective open cohort study. BMJ. 2007;335(7611):136.

17. Hippisley-Cox J, Coupland C, Vinogradova Y, Robson J, Brindle P. Performance of the QRISK cardiovascular risk prediction algorithm in an independent UK sample of patients from general practice: a validation study. Heart. 2008;94(1):34-39.
18. Fiscella K, Fremont AM. Use of geocoding and surname analysis to estimate race and ethnicity. Health Serv Res. 2006;41(4 Pt 4): 1482-1500.

19. Fiscella K, Tancredi D. Socioeconomic status and coronary heart disease risk prediction. JAMA. 2008;300(22):2666-2668.

20. Adler NE, Stewart J. Health disparities across the lifespan: Meaning, methods, and mechanisms. Ann N Y Acad Sci. 2010;1186:5-23.

21. Seeman T, Epel E, Gruenewald T, Karlamangla A, McEwen BS. Socio-economic differentials in peripheral biology: cumulative allostatic load. Ann N Y Acad Sci. 2010;1186:223-239.

22. Franks P, Fiscella K. Reducing disparities downstream: prospects and challenges. J Gen Intern Med. 2008;23(5):672-677.

23. Winters $P$, Tancredi $D$, Fiscella $K$. The role of usual source of care in cholesterol treatment. J Am Board Fam Med. 2010;23(2):179-185.

24. Chang VW, Lauderdale DS. Fundamental cause theory, technological innovation, and health disparities: the case of cholesterol in the era of statins. J Health Soc Behav. 2009;50(3):245-260. 